We appreciate the response to this publication feature and welcome all contributions. Contributions may be sent to José A. Mascorro, our Technical Editor, at his e-mail address: jmascor@tulane.edu. José may also be reached at the Department of Structural and Cellular Biology,

Tulane University Health Sciences Center, 1430 Tulane Ave., New Orleans, LA 70112 and Ph: (504) 584-2747

\section{Cellulose Acetate Replication of Plant Surfaces for SEM}

Gib Ahlstrand

University of Minnesota

ahist007@tc.umn.edu

Replicas of botanical surfaces are sometimes preferred for imaging in the SEM, instead of original surfaces, in cases where it is not possible or desirable, for a variety of reasons, to prepare originals using conventional techniques (chemical fixation followed by dehydration and critical point drying, freeze drying, or cryo-SEM). Samples may be rare (herbarium specimens), or impossible to bring
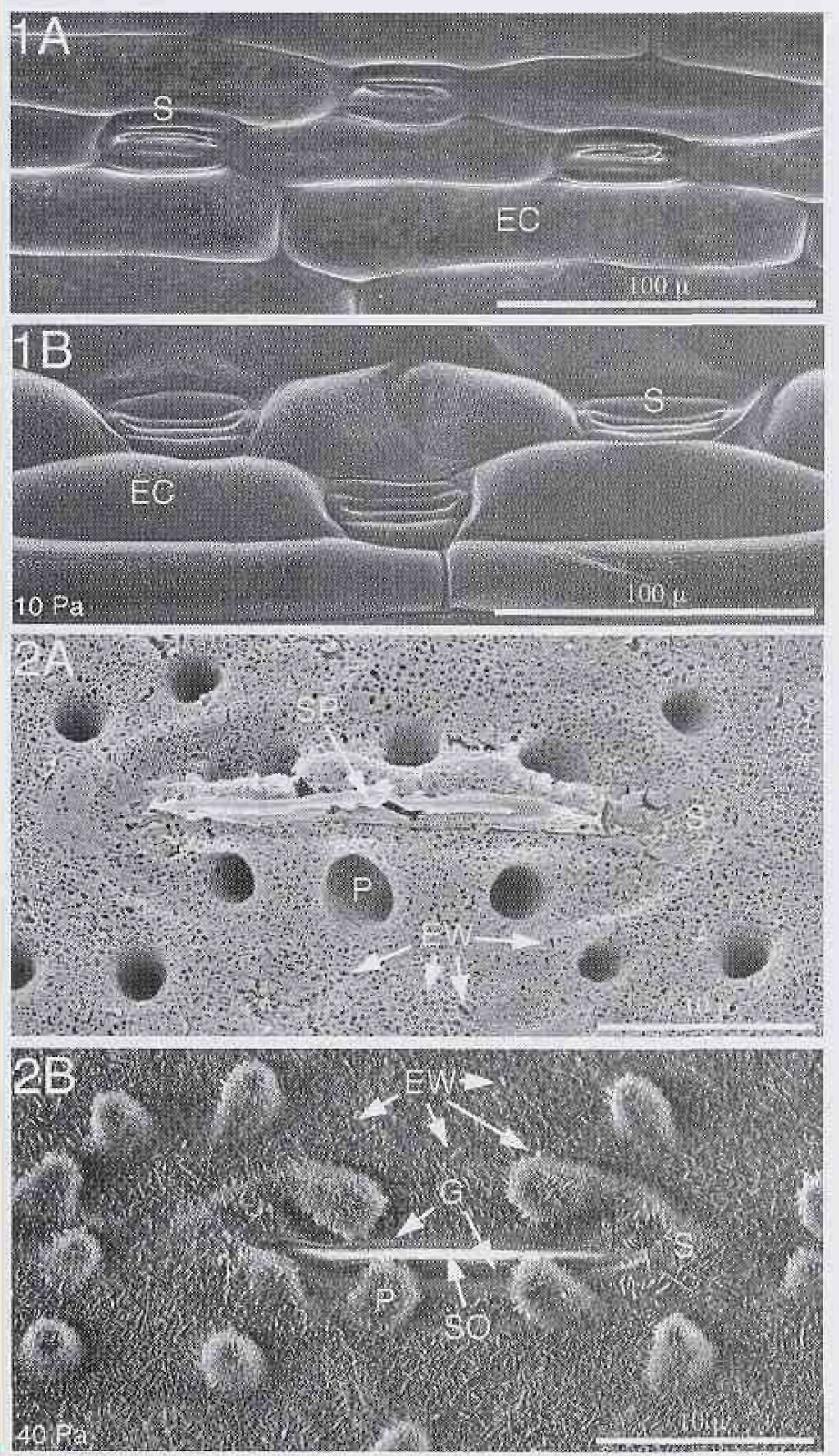

into the lab for processing, and field replication may be the only alternative. Also, the technique is quick and many samples can be easily replicated with a high degree of resolution for surface imaging studies of epidermal cell morphology, epicuticular waxes or distribution of stomata, trichomes, etc., if it is not essential to image directly the original surfaces. The technique could also be used to replicate non-botanical surfaces.

Kay (1) gives an exhaustive compilation of many replicating techniques (most for TEM imaging), and lists references that describe the use of methyl cellulose and ethyl cellulose for replication. North (2) describes the use of cellulose acetate to make stained replicas for imaging plant epidermis using light microscopy. Herein, I describe a method, adapted from North, for making replicas of plant surfaces for SEM imaging using cellulose acetate as the replicating medium.

To make $2-5 \%$ wiv solution (higher concentration yields thicker, more resilient replicas), gradually add $2-5$ grams cellulose acetate to $100 \mathrm{ml}$ dry acetone $(99 \%)$ while stirring on a magnetic mixer. Store in a clean screw capped bottle.

To apply, use clean artist brushes (sizes $1,2,3$ ), either natural or synthetic bristles. Buy new ones and reserve them for this purpose. Rinse brushes in acetone and blot dry immediately after use. Quickly paint the cellulose acetate solution onto the surface to be replicated, typically areas of $0.5-2 \mathrm{~cm}^{2}$. The solution will dry to a white color within seconds. To remove, use forceps to lift the replica off, and place, replica-side up, onto double-stick carbon tape previously mounted onto an SEM stub. Or, very gently apply a $1 \mathrm{~cm}$ length of double-stick carbon tape to the replica, press down very lightly on the tape backing with a cotton swab, or your finger, and then pull up the tape. Carefully peel the tape backing off and mount the assembly, replica side up, onto an SEM sample stub. Gold coat the replica in a sputter coater or vacuum evaporator as usual for SEM specimens.

Figures $1 \mathrm{~A} \& 2 \mathrm{~A}$ show typical replicas made from barley and rice leaves, respectively. Figures $1 \mathrm{~B} \& 2 \mathrm{~B}$ are direct images obtained from the same leaves (nearby areas) imaged in variable pressure mode (VPSEM, 10 \& 40 Pascals pressure, respectively). Epidermal cells $E C$, stomatal structures $S$, papillae $P$ and epicuticular waxes EW (fine texture) are accurately replicated. Care must be taken when determining from a replica if a stoma is open or closed. In Figs. 2 , the stoma opening SO appears in the replica as a plug SP of cellulose acetate. Presumably, if a stoma is closed, the replica would not show a plug but a smooth contour of the guard cells $\mathrm{G}$ meeting. The short rice papillae $P$ replicate well, but longer trichomes on other plant surfaces (not shown) do not replicate easily and will usually leave a hole or a tear in the replica, although they might possibly be counted.

These first impression replicas are the topographical inversion, or "negative", of the true surface, i.e., bumps in the true surface become depressions in the replica. Structures that are tall compared to their width may not replicate and would be represented by a hole in the replica. A "pseudo-positive" image, obtained by inverting the grayscale of the replica image, may be useful in representing the topography of the sample (not shown).

\section{REFERENCES}

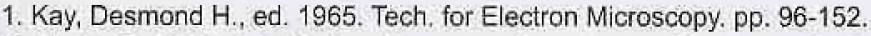

2. North, C. 1956. A technique for measuring structural features of plant epidermis using cellulose acetate films. Nature 178:1186-1187.

\section{ACKNOWLEDGEMENT}

Appreciation for valuable discussions on plant replication is extended to $\mathrm{Dr}$. Richard Zeyen, Dept. Plant Pathology, University of Minnesota. 